\title{
Opciones de mejora cognitiva no convencional como respuesta al desempleo estructural en el contexto tecnológico de la cuarta revolución industrial
}

Unconventional cognitive enhancement options addressing structural unemployment in the technological context of the fourth industrial revolution

\author{
Miguel Moreno Muñoz \\ Profesor Contratado Doctor (acreditado para TU). Departamento de Filosofía II, Universidad de Granada. España \\ mm3@ugr.es
}

\section{TECNOLOGÍAS DE MEJORA HUMANA: DEBATE ÉTICO E IMPACTO SOCIOCULTURAL MONOGRÁFICO COORDINADO POR MIGUEL MORENO (Universidad de Granada) y FRANCISCO LARA (Universidad de Granada)}

\section{RESUMEN}

El objetivo de esta contribución es analizar los efectos en la automatización del trabajo derivados de la implantación de las tecnologías asociadas con la cuarta revolución industrial, y las oportunidades que abren para ciertos programas de mejora cognitiva no convencional. La metodología desarrollada parte de una caracterización del potencial tecnológico asociado con diversas fases del proceso de mecanización y automatización hasta el presente, revisando la literatura que considero imprescindible para entender el potencial diferenciado del ecosistema actual de innovaciones tecnológicas. El interés del enfoque adoptado consiste en integrar tres elementos que suelen encontrarse dispersos en la literatura: 1) el potencial ligado a las tecnologías de la cuarta revolución industrial; 2) diversos análisis del desempleo estructural como fenómeno y tendencia característica de las sociedades contemporáneas; y 3) estudios recientes sobre el progresivo desfase entre desarrollo tecnológico y las dinámicas de adaptación/transformación de los sistemas educativos durante la segunda mitad del siglo XX. Asumo como hipótesis de trabajo que el desfase aludido se ha acentuado como efecto de la digitalización y la incorporación de inteligencia artificial en el desarrollo de sistemas robóticos, cuyas capacidades compiten ya o sobrepasan a las de trabajadores especializados en muchos tipos de actividad. Descartados ciertos proyectos de mejora cognitiva por los riesgos en su actual fase de desarrollo, considero que la evolución de los indicadores de eficiencia de los sistemas educativos públicos y privados durante la última década justifica el interés por determinados programas de mejora cognitiva no convencionales -intento esbozar uno-, como complemento de los programas de reforma en curso.

\section{ABSTRACT}

In this contribution I analyze the effects on the automation of work induced by technologies associated with the fourth industrial revolution, and the opportunities they open for certain programs of unconventional cognitive enhancement. The methodology applied starts with a characterization of the technological potential associated with several stages in the process of mechanization and automation to the present day, reviewing the basic literature in order to better understand the specific potential of the last innovations. This approach allows a more consistent integration of three elements often found scattered in the literature: 1) the potential linked to technologies of the fourth industrial revolution; 2) some studies and reports about the structural unemployment, as a phenomenon and trend that characterizes contemporary societies; and 3) recent studies on the progressive gap between technological development and the dynamics of adaptation/transformation of educational systems during the second half of the twentieth century. As a working hypothesis, I consider that the gap referred has increased as a result of digitization and incorporation of artificial intelligence in the development of robotic systems, in some cases with capacities that compete or exceed those of skilled workers in many activities. Discarded certain attempts of cognitive enhancement for the risks in its current phase of development, I argue that the evolution of indicators of efficiency in public and private educational systems over the last decade justifies the interest in unconventional cognitive enhancement programs -and I try to outline one-, as a complement to the ongoing reforms.

PALABRAS CLAVE

revoluciones industriales | sistema educativo | automatización | digitalización | inteligencia artificial | mejora cognitiva KEYWORDS

industrial revolutions | education system | automation | digitization | artificial intelligence | cognitive enhancement

\section{Introducción *}

Los efectos de la automatización del trabajo en la configuración de la dinámica social y económica contemporánea han sido analizados a partir de una primera generación de máquinas, dispositivos y 
robots industriales que apenas ayudan a comprender el alcance de las transformaciones inducidas por el desarrollo de sistemas robóticos dotados de inteligencia artificial avanzada. En la próxima década, más de 700 tipos de actividades -yacimientos de empleo masivo en los países desarrollados- podrían ser sustituidos por sistemas automatizados capaces de emular o mejorar actividades humanas que requieren capacidades propias de trabajadores con una cualificación media o alta (Frey y Osborne 2013: 2).

El impacto previsible de esta segunda ola de transformaciones sustantivas derivadas de la irrupción masiva de dispositivos conectados a sistemas complejos con gran capacidad de computación, algoritmos especializados y elementos de inteligencia artificial incorporados en su diseño (The Second Machine Age, según la denominan Erik Brynjolfsson y Andrew McAfee 2014) constituye un desafío para las ciencias sociales y para los sistemas educativos de los países económica e industrialmente más desarrollados.

El objetivo de este trabajo es indagar el impacto social de los desarrollos tecnológicos asociados con las tres primeras revoluciones industriales y la transformación experimentada por los sistemas educativos que proporcionaron la cualificación requerida, para identificar los elementos de discontinuidad con los avances de la última década en automatización, diseño de sistemas robóticos, asistentes virtuales y sistemas dotados de inteligencia artificial. Como hipótesis de trabajo se asume un progresivo desfase entre desarrollo tecnológico y transformación/renovación de los sistemas educativos que justifica explorar las posibilidades de un programa de mejora cognitiva no convencional.

\section{Antecedentes}

El progreso en el desarrollo de máquinas capaces de sustituir la aplicación directa de fuerza humana o animal en tareas repetitivas estuvo durante siglos asociado a un potencial limitado para aprovechar fuentes de energía natural fácilmente disponibles (corrientes de agua, viento, mareas, etc.). El propósito de conseguir dispositivos con un funcionamiento fiable sin necesidad de supervisión humana constante contribuyó al desarrollo de sistemas de poleas, resortes, contrapesos y canalizaciones de agua capaces de llevar a cabo tareas relativamente simples, o combinaciones de estas, con aplicaciones de uso militar o civil (incluyendo actividades lúdicas, artísticas o musicales) muy variadas, antes de nuestra era (1).

Entre otras, la eolípila, diseñada por Herón de Alejandría (a quien se atribuye el primer tratado sobre autómatas de la historia) para poner a prueba conocimientos incipientes de hidráulica y mecánica adquiridos a partir del siglo III AC; o el mecanismo de Antiquitera, una especie de computadora analógica con treinta engranajes de bronce, elaborada en el siglo II AC y técnicamente más compleja que cualquier dispositivo construido en el milenio posterior, diseñada para automatizar cálculos astronómicos (Freeth y otros 2006).

La primera revolución industrial, ligada a la producción textil y al empleo de la máquina de vapor, sufrió una transformación radical en 1801, cuando Joseph Marie Jacquard patentó un telar automático programable mediante tarjetas perforadas. Recibido con rechazo por el gremio de tejedores -quemaron públicamente uno de sus telares-, su aproximación a los detalles de una tarea que conocía a la perfección por ser hijo de un obrero textil pronto demostró su potencial. En lo esencial, había diseñado antes que Charles Babbage una primera máquina computacional capaz de automatizar los procesos asociados a una actividad que requería mano de obra intensiva (Delve 2007).

Las sucesivas revoluciones industriales surgieron de una combinación de invenciones, desarrollos tecnológicos y conocimientos científicos que hicieron posible el aprovechamiento de nuevas fuentes de energía (petróleo y electricidad), las propiedades de nuevos materiales (acero, cemento, plástico, silicio) y el diseño de máquinas que transformaron a gran escala la producción industrial, el transporte y las comunicaciones. Las ventajas de la automatización ya eran obvias a finales del siglo XIX (mejora de la calidad y eficiencia en la producción, con un incremento significativo del nivel de vida de gran parte de la población en pocas décadas); pero también sus inconvenientes: reducción de la oferta de trabajo, rigidez en los procesos de ajuste a la evolución de las necesidades, y dependencia de servicios costosos de reparación y mantenimiento, entre otros.

El desarrollo de la electrónica y de la computación digital susceptible de aplicación industrial es un 
fenómeno de la segunda mitad del siglo XX y caracteriza a la tercera revolución industrial. A partir de 1960 se consolida la tendencia a sustituir a trabajadores humanos por sistemas de computación digital en tareas que requerían una cualificación o especialización media, incluyendo muchas dependientes de la información proporcionada por sensores específicos (temperatura, humedad, presión, viscosidad, etc.) para controlar la acción precisa de actuadores muy diversos. Los niveles de control automatizados incluyen al menos uno donde trabajadores humanos monitorizan el funcionamiento y los mensajes de alarma. Estos sistemas están sujetos a una tendencia constante en la automatización de tareas a más niveles, potenciada por las posibilidades de comunicación a escala mundial para transmitir las instrucciones, patrones y diseños de producción a cada factoría o centro de producción (Kottenstette y Antsaklis 2009: 241-244, Vernadat 2009: 1532-1535).

\section{De la robótica industrial a los sistemas robóticos con inteligencia artificial}

La electrónica y la revolución digital han tenido su mayor impacto en la actividad industrial con los robots articulados programables para aplicaciones de montaje, corte y soldadura. Por lo general, se trata de brazos sofisticados con capacidad para articular movimientos en 4, 6 o más ejes (la referencia al respecto son los diseños de KUKA robotics), de distintos tamaños y capaces de adaptarse a tareas específicas (soldadura, pintura, corte, prensado, envasado, apilado y gestión de almacén, tareas en entornos de altas temperaturas, limpieza, etc.), con accionamiento electromecánico. En 1972, FIAT (en Italia) y Nissan (en Japón) comenzaron a disponer de línea de producción con soldadura automatizada (2).

A partir de 1980, las grandes empresas suministradoras de equipamiento industrial en Estados Unidos, Japón, Italia, Suiza y Alemania entraron de lleno en la fabricación de robots articulados para uso industrial (en 1983 ya funcionaban unos 66.000). Se trataba sobre todo de robots diseñados para tareas repetitivas que requerían manipulación muy precisa, aunque las soluciones de automatización se diversificaron rápidamente para ajustarse en la siguiente década a las demandas de trabajo en condiciones muy exigentes: salas limpias, cadenas de producción en la industria del metal controladas íntegramente por sistemas robóticos, entornos que requieren micro-robots actuando de forma coordinada, etc. (Wallen 2008: 11-12).

En 1990 se comienzan a introducir capacidades de comunicación en red que facilitan la coordinación y sincronización del trabajo en la ejecución de procesos industriales. El estándar ISO (ISO 8373:1994,I, equivalente a UNE-EN ISO 8373:1998) define un robot industrial como un manipulador programable en tres o más ejes multipropósito, controlado automáticamente y reprogramable (3). Pero esa definición no proporciona una idea ajustada a la complejidad de los diseños conseguidos: en 1998 ya existían configuraciones de robots industriales dotados de movimiento para desplazarse por entornos cerrados, con brazos cuyo movimiento estaba guiado por láser y capaces de coordinar los movimientos de 27 o más ejes (4). Progresivamente, el uso de robots se extiende fuera del entorno de producción industrial (donde sustituyen a trabajadores humanos en tareas monótonas, repetitivas, pesadas o peligrosas) a tareas como los servicios de seguridad y emergencias, prestación de asistencia sanitaria, exploración científica y extracción minera o de energía, entre otros (Nof 2009: 3-11 y 53-69).

Entre 2000 y 2010 se generaliza el empleo de tecnologías de comunicación inalámbricas, que suprimen los inconvenientes de tener el robot conectado a la unidad de control. Se diseñan robots mucho más eficientes y ligeros, con múltiples sensores integrados y menor consumo energético. Y se les dota con algoritmos que permiten reajustar su funcionamiento para evitar vibraciones o movimientos innecesarios. La digitalización de gran parte de los procesos productivos en todos los sectores de la economía mundial ha permitido niveles de personalización y flexibilidad para reprogramar la producción inimaginables en el siglo XX. Es una tendencia irreversible como factor de competitividad en esta década, pero admite un amplio desarrollo (Brynjolfsson y McAfee 2014: caps. 2 y 4).

La innovación que caracteriza a la cuarta revolución industrial es la incorporación de inteligencia artificial, sensores avanzados y machine learning a los robots y sistemas robóticos (Nof 2009: 249-269). La integración de estos desarrollos con avances en nanotecnología e impresión 3D permite construir sistemas robóticos con diverso grado de autonomía y versatilidad. Además de capacidades básicas de representación de su posición, movimiento y dinámica, muchos pueden procesar información táctil o 
visual y dosificar la fuerza o controlar el movimiento en la trayectoria elegida. Su consolidación va muy ligada al incremento de la capacidad para diseñar sistemas ciberfísicos conectados a una red de servicios descentralizados.

Estos sistemas pueden monitorizar procesos físicos, construir copias o diseños virtuales de un entorno físico determinado y cooperar entre sí, o con seres humanos, para proporcionar una serie de servicios propios o en interacción con otras plataformas de servicios ligadas a una misma cadena de valor. Su flexibilidad les hace capaces de tomar decisiones en tiempo real, dependiendo del análisis de los datos sobre preferencias de los clientes/usuarios que pueden recopilar y procesar mediante algoritmos especializados. El desplazamiento de la capacidad de computación a los centros de datos y el auge de los servicios en la nube, accesibles de forma ubicua mediante redes de comunicaciones con gran ancho de banda, facilita la movilidad de las plataformas de ensamblaje y fomenta modelos de vinculación laboral no presenciales (Velásquez y Nof 2009: 1556-1572).

Si la automatización y la digitalización han contribuido a transformar de modo radical el mercado de trabajo, la integración de robótica con sistemas de inteligencia artificial para gestionar flujos de información, carga de trabajo e incertidumbre en sistemas complejos constituye ahora el principal motor de transformaciones sustantivas en la concepción del trabajo y en la planificación del tiempo, de la formación, del ocio y de la salud (Nau 2009: 249-268, He y Castro-León 2009: 406-414). Las comunicaciones a través de redes digitales de alcance mundial hacen posible la creación de arquitecturas, modelos y metodologías capaces de integrar en tiempo real la información que suministran los consumidores con los procesos de intercambio comercial y las redes de suministro de empresas multinacionales. El nivel de integración y la conectividad de todas las áreas de valor de las redes de suministro ha aumentado significativamente con la llegada del e-Business, e-Commerce, e-Services y la inversión en infraestructura colaborativa para el e-Work en un sistema económico globalizado (Velásquez y Nof 2009: 1556-1572).

\section{Relación entre innovación tecnológica y desempleo estructural}

Cada transformación significativa del sistema productivo ha surgido de un ecosistema de desarrollos tecnológicos cuya integración ha permitido mejorar la eficiencia y la productividad, reduciendo la implicación directa de trabajadores humanos en procesos repetitivos y fácilmente automatizables. Las ventajas de la automatización incluyen una reducción de la accidentalidad y de los riesgos laborales (Morel y otros 2009: 738-745), aumento de precisión y calidad en el producto, reducción de costes ligados al mantenimiento y mejora de la productividad.

Sus contrapartidas han sido objeto de intenso debate desde que Jacquard presentó su telar automático a los patrones de la industria textil de la época (Autor 2015: 3-9). La principal amenaza directa en las revoluciones previas ha sido la destrucción de millones de puestos de trabajo en determinados sectores susceptibles de automatización (logística, manufactura y transporte, p. ej.), ligada a una distribución muy desigual de los beneficios derivados del incremento de productividad (Daunton 1995: 377-476).

Si bien un porcentaje importante del desempleo asociable con la innovación tecnológica podría considerarse compensado mediante la aparición de nuevos yacimientos de empleo y la demanda de mayor cualificación para los operarios en entornos productivos muy automatizados, con un impacto directo en la mejora de las condiciones salariales, los temores a un balance neto desfavorable como efecto de la mecanización y de la automatización han suscitado reacciones airadas en innumerables ocasiones (desde los movimientos luditas hasta las huelgas masivas en el sector del metal, p. ej.) y continúan siendo motivo de inestabilidad política o conflicto social (Jones 2006).

En su informe de enero de 2016, la OIT (Organización Internacional del Trabajo) calculaba el desempleo mundial en 197,1 millones de personas, 27 millones más que antes de la crisis de 2007 (5). Entre los factores causales con impacto a escala mundial no menciona la innovación tecnológica, sino el descenso de la inversión de capital a largo plazo, la ralentización del crecimiento de la población en edad laboral y una distribución desigual de los beneficios derivados del crecimiento (puesto que después de la crisis los ingresos del 1 por ciento más rico crecieron a un ritmo mucho mayor que los del resto de la población). 
Sus previsiones para los próximos dos años son pesimistas: el número de desempleados a nivel mundial se incrementará en 2,3 millones en el año 2016, y en 1,1 millones en 2017, afectando en su mayor parte a las economías emergentes. Por tratarse de niveles de desempleo generalizado muy altos entre la población joven de las economías desarrolladas, la OIT lo considera un factor determinante de malestar social, con claro reflejo en los movimientos políticos y sociales (ibid.: 17, gráfico 9) (6).

En 2015 la OIT sí tuvo en cuenta el impacto de la automatización y la innovación tecnológica en el desempleo de larga duración, con efectos sobre un número creciente de trabajadores cualificados:

"Algunos de estos acontecimientos obedecen a la reducción durante los últimos años de los puestos de trabajo rutinarios que requieren calificaciones medias. Esto se ha producido paralelamente a la demanda creciente de puestos de trabajo en los extremos superior e inferior de la escala de calificaciones. En consecuencia, trabajadores relativamente formados que solían ocupar estos puestos de trabajo que requerían calificaciones medias, se ven ahora cada vez más obligados a competir por ocupaciones que requieren pocas calificaciones. Estos cambios ocupacionales han configurado pautas de empleo y también han contribuido a ampliar la desigualdad en los ingresos registrada durante los últimos decenios" (OIT 2015: 4).

Las valoraciones pesimistas y optimistas del impacto de la tecnología en el desempleo estructural se presentan muy condicionadas por el manejo de versiones a corto o largo plazo del fenómeno, combinadas con otros estudios sobre tendencias demográficas y socioeconómicas (Danaher 2016: 4-6). El análisis prospectivo riguroso resulta de vital importancia para identificar las disfunciones y reducir en lo posible el coste de los procesos de ajuste para empresas, individuos y sectores económicos o sociedades en su conjunto.

Economistas inequívocamente optimistas como David H. Autor y John Danaher (para quienes el desempleo tecnológico no ha causado ninguna crisis general de la economía a largo plazo porque los trabajadores desplazados -o al menos las generaciones siguientes- han encontrado otras formas de trabajo) se muestran preocupados por la dimensión que ha alcanzado en los últimos años el desempleo estructural, como indicador de que el ritmo acelerado de innovación tecnológica parece haber sobrepasado las capacidades de generaciones sucesivas de trabajadores para adquirir el tipo de formación que les permitiría encontrar formas alternativas de trabajo (Autor 2015: 22-27, Danaher 2016: 3-6).

\section{El fin del trabajo y los ciclos de obsolescencia en el capital humano}

En el capítulo 12 de su obra The end of work (titulado "Requiem for the Working Class", pp. 181-197), Jeremy Rifkin advertía de la gravedad de los riesgos para el empleo y para toda la dinámica social que entrañaba el proceso de automatización acelerada en la década de los noventa. A diferencia de las dos primeras revoluciones industriales, el objetivo de la tercera no era sustituir solo el esfuerzo físico del trabajador humano por sistemas mecánicos más o menos automatizados, sino integrar los desarrollos tecnológicos de la Era de la Información (computadoras en red, robots y sistemas con capacidad de computación en paralelo y algoritmos de inteligencia artificial) para desplazar también a gran parte de los trabajadores de cuello blanco con cualificación multinivel.

Según sus estimaciones, el desempleo derivado del uso de máquinas inteligentes podría afectar al $75 \%$ de la masa laboral en los países industrializados y poner en riesgo la viabilidad de 90 millones de puestos de trabajo, sobre un total estimado de 124 millones (Rifkin 1995: 5). La combinación de automatización inteligente y desarrollos biotecnológicos podía afectar de modo drástico a la mitad de la población humana, vinculada a labores de agricultura y ganadería; pero también a buena parte de la dedicada al sector servicios (Rifkin 1995: 109 y 143).

Sus conclusiones quizá resultan hoy algo prematuras por el retraso en la difusión de ordenadores capaces de entender el habla, leer texto escrito y llevar a cabo tareas de gestión o cálculo desempeñadas antes por seres humanos (Rifkin 1995: 136-143). Pero es evidente que la precarización del empleo y el desempleo estructural (el causado "no por falta de demanda, sino por cambios en los patrones de demanda o la obsolescencia de la tecnología, que requieren reconversión de los 
trabajadores y grandes inversiones en nuevos bienes de capital") (7) constituye un desafío a escala planetaria que afecta -aunque de modo desigual- a trabajadores con niveles de cualificación muy diversos, incluyendo a titulados superiores, profesiones liberales y a personal con experiencia en puestos de dirección (World Economic Forum 2016: 5).

Dos décadas después de su análisis, las capacidades avanzadas de comunicación y automatización inteligente que pronosticaba Rifkin se han incorporado a dispositivos móviles al alcance de cualquier consumidor y exhiben un claro potencial para tareas cualificadas que ponen en peligro millones de puestos de trabajo en el sector público y privado (administración, aseguradoras, banca, logística y distribución al por mayor, servicio postal, transporte, seguridad, etc.).

Para Jeremy Rifkin (en sintonía variable con autores como N. Carr, M. Ford, J. Kaplan, E. Brynjolfsson y A. McAfee) (8) una época está llegando a su fin. Ante los problemas derivados de la eliminación de puestos de trabajo y el consiguiente paro no hay respuestas por parte de la teoría tradicional del capitalismo industrial: las instituciones básicas, públicas o privadas, de la sociedad actual (Estado, sindicatos, sistema educativo) se muestran incapaces de responder con eficacia a un desempleo estructural persistente. Tiene sentido, pues, plantearse una reconfiguración del orden social sobre nuevas reglas de juego (un nuevo contrato social) que afronten el reparto inevitable del trabajo asalariado, la reducción de la semana/jornada laboral, la potenciación del voluntariado, la universalización de una renta básica y la orientación del sistema fiscal para promover la economía social (cfr. Rifkin, ibid.: 221-293).

\section{Desafíos para la formación y el empleo en el siglo XXI}

El análisis prospectivo de Rifkin no aborda de manera satisfactoria los ajustes requeridos por los sistemas educativos de los países desarrollados para adaptar la formación de las generaciones futuras de trabajadores a la evolución acelerada del cambio tecnológico en su transición a la cuarta revolución industrial. Se limita a reconocer la baja calidad de los programas federales de reciclaje (Rifkin 1995: 50), el bajo nivel de alfabetización entre la clase trabajadora estadounidense (Rifkin 1995: 37) y la brecha entre la cualificación requerida para los empleos bien remunerados y la cualificación máxima a la que podían aspirar la mayoría de los trabajadores inscritos en programas de reentrenamiento (Rifkin 1995: 36). Un escenario dramático, puesto que los trabajadores con baja cualificación vinculados a actividades en entorno urbano vieron reducido su salario un 14\% en el período $1973-1989$ (hasta un $24 \%$ entre la población afroamericana).

Autores como N. Agar (9) y R. Kurzweil (10) cuestionan lo que Danaher denomina falacia ludita (la creencia en la capacidad de una generación de trabajadores para seguir formándose y readaptarse profesionalmente a una velocidad igual o mayor que el ritmo de innovación tecnológica). El incremento en la eficacia de la cooperación social resulta especialmente visible en el desarrollo de la investigación científica de naturaleza interdisciplinar y en el acortamiento de los períodos para su aplicación tecnológica en los sectores estratégicos de actividad económica. Pero es evidente que las instituciones educativas responsables de capacitar para un empleo cualificado a la mayor parte de la población en los países desarrollados se han visto sobrepasadas por la aceleración en los ciclos de innovación tecnológica y el lastre de múltiples disfunciones internas: masificación, inercia burocrática, infrafinanciación crónica, sistemas de incentivos que favorecen la mediocridad y una distribución injusta de privilegios no ligados al mérito como la edad o la sumisión a los vaivenes normativos, entre otras (Guereña y Tiana 1989, García 1995, Tiana y otros 1996: 73-74, Ginsberg 2013: 65-96).

La desorientación en aspectos tan decisivos como los sistemas de gobernanza en todos los niveles del sistema educativo (Gerber 2014) ha provocado una hipertrofia de la tareas administrativas en detrimento de la calidad de los empleos relacionados con la docencia y la investigación (Ginsberg 2013: caps. 1, 3 y 5). El debate sobre los criterios de calidad y la metodología para evaluar el rendimiento de estudiantes, personal docente y facultades o centros se sustenta en presupuestos tan subjetivos e ideologizados como hace 20 años (Tiana y otros 1996, Rey 2010, Wolte 2010). El incremento de las tasas de fracaso (en la educación obligatoria) y de abandono temprano (en la etapa postobligatoria) ha adquirido proporciones tan preocupantes $-30 \%$ de promedio en España- que pone en riesgo el modelo social dentro y fuera del contexto europeo (Cobo 2010, Rué 2014, García y Adrogué 2015). 
En conjunto, las debilidades y disfunciones de los sistemas educativos públicos y privados en muchos países desarrollados consumen los recursos que deberían destinarse a incrementar su eficacia como instancia inclusiva y generadora de oportunidades (Au 2008, Smith 2008). Esto sucede en un sistema económico globalizado que cada año precariza más el empleo y otorga menos valor tanto a la formación profesional como al título universitario (Normand 2008, Bertocchi y Spagat 2004), con retrocesos tan llamativos como el auge de la contratación, por parte de las élites empresariales y para los segmentos salariales más altos, dentro de su mismo círculo social (Krug 2012, Chua 2011, Bähr y Abraham 2016, Bian y Huang 2015).

La retórica del emprendimiento y la orientación empresarial en las reformas de la educación secundaria durante la última década han ido en paralelo a la desnaturalización de las universidades y de su función social (Newfield 2008, Maher y Tetreault 2008, Donoghue 2008), sometidas a fuertes tensiones por efecto de los cambios acelerados en el sistema económico y el aumento de los costes de funcionamiento. Las nuevas demandas de cualificación para una sociedad cada vez más desigual, con niveles crecientes de desempleo estructural -que imponen ciclos acelerados de reciclaje profesional en cualquier etapa de la vida activa- han puesto en evidencia las insuficiencias de los sistemas educativos consolidados a lo largo del siglo XX y el escenario de incertidumbre y desconcierto desde el que se afrontan los nuevos desafíos (Krueger y Kumar 2004, Bozsik 2015, Berg y Seeber 2016).

Cuando un nuevo campo disciplinar adquiere importancia estratégica como generador o "atractor" de valor económico, la mayor parte de las universidades y centros de investigación se ven incapaces de proporcionar salarios competitivos para retener el talento y beneficiarse de la innovación. Durante los años ochenta y noventa, con la mayor parte de los expertos trabajando en las universidades, el campo de la inteligencia artificial estaba tan desatendido e infrafinanciado como el grueso de la investigación básica no ligada a grandes infraestructuras. En la actualidad, los responsables de los centros de ingeniería computacional, robótica, machine learning e inteligencia artificial ni siquiera consiguen retener a los estudiantes de grado, tentados por las ofertas de las grandes empresas del sector incluso antes de terminar (11).

\section{La dinámica recombinante de la cuarta revolución industrial}

La digitalización y el incremento conforme a la Ley de Moore en la capacidad de computación, combinados con el aumento de la velocidad de los flujos de información y la reducción de costes como efecto de la automatización, contrastan con el período relativamente estable de 25-30 años que se requiere para educar y formar a cualquier trabajador especializado. Resulta verosímil considerar que los estudios sobre el desempleo estructural apenas disponen de perspectiva para observar la porción relativamente lineal de una curva de crecimiento de los avances tecnológicos que resulta ser exponencial (Danaher 2016: 5, Brynjolfsson y McAfee 2014: caps. 3, 4 y 6).

Para Brynjolfsson y McAfee, el carácter recombinante de las mejoras en computación, digitalización y automatización, potenciado por la incorporación de inteligencia artificial y nuevas técnicas de producción deslocalizada mediante impresión en 3D, no constituye el logro más destacable de la era del computador: se trata de simples ejercicios de calentamiento. La auténtica revolución, sin precedentes en la historia, es el desarrollo de sistemas con inteligencia artificial y la interconexión de la mayor parte de la población humana a través de una red digital común.

Cualquiera de estos avances habría inducido transformaciones profundas en las perspectivas de crecimiento, considerados aisladamente. Combinados, dan lugar al fenómeno con mayor potencial para transformar el trabajo físico e intelectual desde la revolución industrial. La falta de perspectiva sobre el desempleo estructural en la nueva fase de desarrollo tecnológico distorsiona la comprensión de lo que puede suceder cuando esta combinación de avances tecnológicos realmente comience a despegar (Brynjolfsson y McAfee 2014: 89).

La cruzada por la calidad y la excelencia en la universidad, cuyo último envite ha empantanado en un lodazal burocrático y administrativo los últimos reductos de actividad profesional con potencial para aportar innovación y formación cualificada a quienes el desempleo estructural devuelve al sistema educativo, tuvo sus precedentes en la carga contra los maestros e instituciones educativas 
estadounidenses en los años noventa. En lugar de denunciar la infrafinanciación crónica y el desfase tecnológico en la infraestructura educativa para hacer frente con solvencia a demandas sin precedentes, revistas y periódicos enfatizaban la ineficiencia y su contribución al deterioro de la calidad de vida de la próxima generación de trabajadores (Rifkin 1995: 51).

\section{La revolución pendiente en los sistemas educativos}

Asumiendo un amplio margen de incertidumbre, tanto en la perspectiva como en la prospectiva a medio plazo sobre el impacto económico y social de las tecnologías impulsoras de la cuarta revolución industrial, considero el enfoque de Brynjolfsson y McAfee acertado en sus tesis fundamentales. En particular, las referidas a la rigidez e insuficiencia de los sistemas educativos públicos y privados para proporcionar la formación adecuada a trabajadores sometidos a dinámicas de cambio sociotécnico acelerado, en el contexto inestable y complejo de las economías globalizadas (Goldin y Katz 2010: 7-8 y 227-244).

Si bien existe la posibilidad de que los desarrollos tecnológicos -por sorprendentes que resulten- puedan integrarse de modo que complementen las habilidades, conocimientos y competencias profesionales proporcionados por los sistemas educativos de los países desarrollados a las próximas generaciones de trabajadores, considero más probable un escenario donde las restricciones presupuestarias, las inercias burocráticas y el desplazamiento del foco de interés hacia otras cuestiones urgentes (problemas ambientales, amenazas a la seguridad, movimientos demográficos, enfermedades emergentes, competencia por recursos naturales y fuentes de energía, etc.) agudizarán el desfase entre sistema educativo y mercado laboral.

Este desfase pudo pasar desapercibido en la primera mitad del siglo XX, pero no en la segunda, como muestran Goldin y Katz (2010: 290). Cuando analizan (cap. 8) los retornos de la educación bajo el esquema conceptual de una carrera entre la educación (el suministro de habilidad) y los cambios tecnológicos (la demanda de habilidad), observan cómo ha variado el concepto de trabajador cualificado a lo largo del siglo pasado: hasta 1950 significaba haber finalizado la enseñanza secundaria (o formación profesional de ciclo medio); entre 1950 y 2005 se considera cualificado al profesional con formación de grado o posgrado universitario.

Si en esta carrera la educación avanzó más rápido que la tecnología durante la primera mitad del siglo, en los últimos 30 años la tecnología se mantuvo a la cabeza, pese a que en ambos períodos eran constantes los cambios tecnológicos que demandaban nuevos expertos. Goldin y Katz (2010: 303) no atribuyen este desfase a una aceleración del cambio técnico que exige conocimientos especializados, sino a la falta de crecimiento del sector educativo. Pero en este aspecto su análisis resulta insatisfactorio: no consigue explicar el desfase entre los salarios más altos (se disparan) y los medios-bajos (apenas aumentan) de un amplio grupo de trabajadores altamente cualificados. Atribuyen esta desigualdad a una inversión insuficiente en educación, que impide a muchas familias asumir el coste de la formación superior.

Con algo más de perspectiva sobre las dos últimas décadas, Thomas Piketty aporta un análisis mucho más sutil del fenómeno: se puede constatar el aumento de la brecha salarial entre graduados universitarios y quienes no van más allá de la escuela secundaria, como muestran Goldin y Katz. Pero esto va ligado a un fenómeno muy específico: el 1\% (y, aún más, la parte superior del 0,1\%) del grupo con graduación universitaria han visto despegar sus salarios frente al resto con formación equiparable. Esta desigualdad se constata incluso entre grupos relativamente homogéneos que realizaron sus estudios en las universidades de élite (Piketty 2014: cap. 9, "Inequality of Labor Income").

En cuanto a participación en la renta nacional, el segundo fenómeno es el relevante: la sobreactuación del percentil superior explica el grueso (casi tres cuartas partes) del aumento de la participación del decil superior en la renta nacional de Estados Unidos desde 1970. Piketty considera importante hallar una explicación satisfactoria para este fenómeno, y descarta que merezca la pena buscarla en el factor educativo (12).

En mi opinión, Piketty aporta los datos que faltaban para identificar un factor clave: el proceso de 
aceleración inducido por las innovaciones tecnológicas en robótica e inteligencia artificial, combinadas con otras (automatización, servicios en la nube, big data, impresión 3D, supercomputación, etc.) que suelen asociarse con el núcleo de la cuarta revolución industrial (Dirican 2015). Son las habilidades para sacar provecho de estos desarrollos tecnológicos avanzados las que determinan el valor de los profesionales que sólo las mejores empresas pueden permitirse contratar (cfr. nota 17).

En países como España, con capacidad tecnológica pero alejados del nivel y ritmo de innovación que caracteriza al sistema productivo estadounidense, la inclusión de las prestaciones en educación son un elemento fundamental para reducir las desigualdades. Sin embargo, las administraciones públicas no han logrado revertir la tendencia al aumento en las desigualdades iniciada en 2007. La educación secundaria y la formación profesional (obligatoria entre 12 y 15 años), han tenido un efecto redistributivo importante en la renta (en torno a 1,5 puntos de Gini de reducción), mientras que la educación universitaria apenas reduce el índice de Gini, cuando este gasto es añadido a la renta disponible de los hogares (Goerlich 2016: 145 y 154-168).

Las importantes reducciones del gasto público en sanidad y educación, como efecto de las políticas de consolidación fiscal en el período recesivo posterior a 2007, permiten entender en parte por qué los efectos de la educación superior son más inciertos y, en general, tienden a mostrar un comportamiento regresivo en la reducción de la desigualdad: en períodos de crisis y desaceleración económica se requiere un apoyo proporcionalmente mucho mayor para compensar el ritmo acelerado de innovaciones en los sectores estratégicos $\underline{(13)}$.

\section{Explorando opciones no convencionales}

\subsection{Alfabetización múltiple y mejora cognitiva tecnológicamente asistida}

En The element: how finding your passion changes everything, Ken Robinson y Lou Aronica articulan un relato convincente sobre la rigidez de los sistemas educativos diseñados para las tres primeras revoluciones industriales (Robinson y Aronica 2009: 27-82). Con una estructura similar en la oferta y jerarquización de contenidos, dificultan el desarrollo de la creatividad y excluyen muchos tipos de talento que serán necesarios para el florecimiento de la cultura sofisticada, las artes, la innovación científicotécnica y el pensamiento crítico en los próximos años.

Muy pocos sistemas educativos consiguen dotarse -en cualquiera de sus niveles- de la infraestructura necesaria para conseguir que los estudiantes con más talento encuentren en ellos su elemento, es decir, un contexto para llevar a cabo actividades de aprendizaje estimulantes, con los medios materiales y el soporte económico necesario para desarrollar sus múltiples habilidades y dar proyección profesional a su creatividad e imaginación (Robinson y Aronica 2009: cap. 1, “The Element”).

De hecho, el porcentaje de personas que no terminan sus estudios universitarios, en países como Estados Unidos o España, es extraordinariamente alto (un 30\%, como promedio global; y cercano al 50\% en algunas titulaciones de Humanidades y Ciencias Sociales). El nivel de insatisfacción entre estudiantes, profesores y padres es muy elevado, sobre todo referido a los primeros cursos de grado. Desde el punto de vista institucional, se considera un objetivo ambicioso reducir el abandono escolar a menos del 10\% (el promedio en la Unión Europea está sobre el 15 por ciento), y conseguir que al menos el $40 \%$ de la población entre 25 y 35 años consiga formación superior (14).

Aunque el abandono de los estudios universitarios es un fenómeno complejo, que involucra factores externos (situación económica, contexto socio-cultural de procedencia, etc.) y condicionantes individuales (expectativas, capacidad de adaptación, determinación, motivación y habilidades para gestionar el tiempo, entre otras), para Robinson y Aronica es un indicio claro -junto a la devaluación de los títulos universitarios- de que cada vez más, la estructura y el carácter de la industria educativa chirrían bajo la tensión del siglo XXI (Robinson y Aronica 2009: cap. 11, "Making the Grade").

De poca utilidad resultan los enfoques convencionales ante un desafío de estas características. Es necesario explorar variantes más imaginativas que los miles de intentos reformistas conocidos. Y en este punto es donde mi aportación conecta con las tecnologías de mejora cognitiva, que otros colegas 
abordan bajo diferentes perspectivas en este mismo volumen.

Las acciones de mejora convencionales (reformas normativas, renovación de los criterios psicopedagógicos, adecuación de la infraestructura educativa, reducción del tamaño de los grupos, aumento de la cualificación del profesorado, diversificación curricular, etc.) pueden complementarse con otras inspiradas en ciertas líneas de mejora cognitiva orientadas a la alfabetización múltiple (multiliteracy), asistidas por una infraestructura inteligente de informática y telemática educativa centrada más en los procesos, intereses y habilidades de cada estudiante que en los resultados (15).

Por mejora cognitiva entiendo la amplificación o la extensión de las capacidades básicas de la mente, mediante el aumento o la optimización de nuestros sistemas de procesamiento de información. Según Anders Sandberg, la cognición consiste en los procesos que un organismo utiliza para organizar la información: percepción, selección (atención), representación (entendimiento) y retención (memoria); y, sobre esa base, orientar el comportamiento y la respuesta motora (Sandberg 2011: 71).

Aunque la literatura sobre mejora cognitiva de la última década ha prestado mucha atención a las intervenciones genéticas, farmacológicas o neurobiológicas destinadas a perfeccionar diversos aspectos de la biología humana asociados con la memoria, los procesos de atención y el control emocional, entre otros $\underline{(16)}$, me interesan aquí las tecnologías, servicios y aplicaciones con un potencial contrastado para la misma finalidad que no están sujetas a debate ético por sus posibles efectos indeseados ni son vulnerables a lo que denomino la objeción distópica (el tipo de objeciones que suscitarían, p. ej., estrategias de control social sustentadas en un empleo masivo de psicofármacos o de sistemas de vigilancia con implantes nanoelectrónicos mediante coerción).

Las intervenciones sociales y educativas convencionales con potencial para mejorar la función cognitiva relacionada con las facultades básicas pueden amplificarse extraordinariamente gracias a la convergencia de desarrollos en informática, tecnologías de la información e inteligencia artificial. Existen miles de dispositivos cuyo hardware y software interno se ha convertido ya en el medio para acceder a servicios y contenidos externos que millones de seres humanos demandan como extensión natural de sus facultades biológicas o adquiridas. Los usuarios de esos dispositivos se benefician rutinariamente de servicios y aplicaciones capaces de potenciar algún subsistema cognitivo implicado en tareas de cálculo, lenguaje o visualización (sin necesidad de corregir patologías o disfunciones específicas), por lo que en términos estrictos cabe considerarlos dispositivos de mejora cognitiva no convencional (cfr. Sandberg 2011: 72-76) (17).

\subsection{Infraestructura tecnológica de e-learning al servicio de la cooperación social}

La eficacia del esfuerzo colectivo de cooperación orientado a facilitar el acceso al conocimiento y a la cultura se ha visto extraordinariamente amplificada en las sociedades con infraestructura avanzada de comunicaciones. Además de los sistemas reglados de acceso al conocimiento, existen múltiples fuentes de recursos, servicios y aplicaciones con claro potencial para reforzar, mejorar y diversificar los procesos de aprendizaje de los estudiantes en cualquier etapa del sistema educativo. La cuestión es cómo aprovechar todo este potencial mediante las herramientas adecuadas, y conseguir su integración en un programa de mejora cognitiva no convencional, a la medida de las capacidades, ritmos, grados de autonomía e intereses individuales que pueden orientar las selección de recursos.

La reducción de costes en los dispositivos de ocio y comunicación más demandados pone al alcance de millones de usuarios de cualquier estrato socioeconómico aplicaciones y servicios con enorme potencial para la mejora cognitiva tecnológicamente asistida. La computación ubicua y la convergencia de todos los soportes de información en el formato digital es una revolución insuficientemente aprovechada por los sistemas educativos. Puede contribuir a desarrollar programas de alfabetización múltiple (texto/audio /visual/digital), de estímulo del pensamiento crítico -en ciencias, artes y humanidades-, y de la capacidad para interaccionar y comunicarse con objetivos mucho más ambiciosos que los subyacentes a la rígida oferta de itinerarios formativos en los sistemas públicos y privados (Halinen y otros 2015: 140-147).

El desarrollo de las aplicaciones conectadas a servicios en la nube, ofrecidos a través de asistentes virtuales, plataformas o dispositivos que aprovechan los desarrollos en inteligencia artificial y el ancho de banda para suministrar productos de ocio y entretenimiento personalizados, tienen un enorme potencial para potenciar procesos de aprendizaje en usuarios con perfiles educativos y profesionales muy 
diversos, según su edad, etapa educativa, habilidades previas y grado de especialización. Pero su materialización requiere una apuesta decidida por actualizar y renovar la infraestructura educativa y mantener la inversión en capital humano -aspectos seriamente afectados en muchos países a partir de 2007, coincidiendo con la gran recesión- (Brynjolfsson y McAfee 2014: cap. 4, pp. 109-126).

La educación superior ha incorporado con éxito, en las dos últimas décadas, plataformas de teleformación (e-learning) que complementan y potencian (incluso podrían sustituir) a casi todos los procesos de enseñanza y aprendizaje presenciales. Moodle quizás sea la más popular; pero existen otras muchas (Sakai, Dokeos, Claroline, Chamilo...) y servicios o aplicaciones que incorporan funciones básicas similares (Google Clasroom, Schoology, p. ej.). Lo esencial es que incorporan herramientas de gestión del aprendizaje compatibles con un amplio rango de criterios de intervención psicopedagógica, metodologías de trabajo y seguimiento autónomo, sobre recursos electrónicos propios o accesibles mediante suscripción (18).

La masificación de los grupos (35-40 estudiantes por aula en secundaria; 85-100 en los estudios de grado de universidades españolas) dificulta extraordinariamente aplicar metodologías de aprendizaje individualizado. Evitar esto es, al fin y al cabo, una cuestión de prioridades, incluso en los ciclos económicos menos favorables. Pero, si la infraestructura tecnológica es la adecuada -y los costes de acceso razonables-, la masificación no impide que muchos estudiantes puedan seguir itinerarios no presenciales bastante personalizados: basta aplicar un criterio profesional solvente para elegir la secuenciación apropiada de actividades y ofrecer una selección de recursos complementarios sobre los que desarrollar la curiosidad individual, más allá de los objetivos del curso o de lo exigido en las pruebas de evaluación externa.

\subsection{La mediación de asistentes virtuales en el acceso a plataformas de recursos educativos}

La posibilidad de interacción entre asistentes virtuales como Siri, Google Now, Alexa o Cortana (19) y plataformas de teleformación como Moodle o las que gestionan la creciente oferta de cursos en abierto (Coursera, edX, Miriada X), diseñados para un seguimiento masivo (MOOC: Massive Open Online Course) $(20)$, puede revolucionar la eficacia de los sistemas educativos en los próximos años.

Además de ampliar las opciones sobre cualquier proceso de aprendizaje en cualquier etapa, su diseño tiende a simplificar el seguimiento de contenidos hasta hace poco al alcance de estudiantes inscritos en los centros e instituciones más elitistas (entre las impulsoras de los MOOC se encuentran el MIT, Harvard, Stanford o Yale) (21).

Las bases de datos con recursos educativos digitalizados y en abierto (la Khan Academy es un ejemplo interesante) (22) se incrementan continuamente, gracias a la cooperación -casi siempre desinteresadade miles de usuarios y profesionales que consideran importante ponerlos a disposición del mayor número de personas posible, bien participando en el diseño o bien traduciendo y adaptando a lenguas y contextos muy diversos.

Las herramientas de traducción automática y subtitulado de vídeos educativos han mejorado de modo radical la accesibilidad a contenidos de calidad para millones de usuarios. Este proceso continúa ampliándose a medida que las aplicaciones y servicios se dotan de mejores sistemas de procesamiento del lenguaje natural y facilitan modos mucho más naturales de interacción, para una base de usuarios que crece sin parar.

\section{Conclusión}

El potencial de la creatividad humana al servicio de la cooperación social para facilitar el acceso al conocimiento y a la educación parece ilimitado. Durante la primera mitad del siglo XX ese esfuerzo produjo unos resultados extraordinarios, creando riqueza y contribuyendo al progreso social en una escala sin precedentes. Pero existen razones para sostener que los sistemas educativos no han evolucionado en las últimas décadas al ritmo impuesto por la digitalización, la automatización y las tecnologías de la cuarta revolución industrial.

El desfase entre el tipo de innovación tecnológica aplicada en la industria de productos o servicios con 
mayor valor añadido y la lentitud de los procesos de actualización de la infraestructura tecnológica en los sistemas educativos resulta agravado por la reducción de las inversiones en capital humano que siguieron a la gran recesión. Las limitaciones de los sistemas de educación presencial, masificados y costosos, invitan a explorar propuestas de mejora cognitiva no convencionales, entre las cuales parece obligado preferir aquellas que no plantean obstáculos de tipo ético (Ansari 2015).

Mi propuesta se sustenta en la integración de tecnologías que, por separado, ya son relativamente bien conocidas o están en proceso de serlo. La integración de desarrollos en tecnologías de la información, plataformas de teleformación y asistentes virtuales conectados a sistemas dotados de inteligencia artificial avanzada puede contribuir a la aparición de nuevos sistemas de aprendizaje, compatibles con una variedad de criterios metodológicos, intereses individuales y niveles de asistencia personalizada sin precedentes, sobre repositorios y bases de recursos en continua expansión.

La posibilidad de integrar en los sistemas educativos servicios, aplicaciones y asistentes informáticos complejos, dotados de la capacidad de aprender y de generar formas propias de creatividad, no sólo de potenciar la humana, es un componente de la cuarta revolución industrial con gran potencial para contribuir a reducir el desfase entre sistema educativo y desarrollo tecnológico. Es poco probable que la capacidad humana de inventiva (ideación creativa, a partir de datos o información previa cada vez más fácil de obtener) y las habilidades para reconocer patrones complejos o para establecer formas sofisticadas de comunicación puedan ser emuladas, con un potencial equivalente, en sistemas informáticos dotados de inteligencia artificial (Brynjolfsson y McAfee 2014: 375).

Las distopías tecnológicas tienen en el desempleo estructural un fuerte aliado (Di Salvo 2013, Giles 2013). Pero la última década proporciona notables ejemplos del potencial humano para crear valor y originar nuevas categorías de productos, ecosistemas de servicios e incluso ramas de actividad industrial, combinando el potencial de la digitalización, de la robótica y de los desarrollos en inteligencia artificial con el talento humano.

Los sistemas educativos necesitan una profunda reforma y actualización de su infraestructura tecnológica para mantener su protagonismo en la preparación de las nuevas generaciones de profesionales y trabajadores expertos. La inversión en capital humano debe dimensionarse para evitar los efectos negativos de la masificación, explorando múltiples modos -convencionales o no- de combinar el potencial de las máquinas y sistemas inteligentes con las habilidades humanas para la cooperación y el aprendizaje.

* Este trabajo ha contado con el apoyo del proyecto FFI2012-32565: Ética y política de los avances biomédicos en mejora humana. Convocatoria 2012, subprograma de Proyectos de investigación fundamental no orientada.

\section{Notas}

1. Cfr. las entradas Automaton, Aeolipile y Herón de Alejandría en: https://en.wikipedia.org /wiki/Automaton; https://en.wikipedia.org/wiki/Aeolipile;

https://en.wikipedia.org/wiki/Hero of Alexandria.

2. Cfr. http://www.ifr.org/history y https://www.robots.com/education/industrial-history.

3. Cfr. http://www.aenor.es/aenor/normas/normas/fichanorma.asp?tipo=N\&codigo=N0013713.

4. Cfr. https://robotics-vo.us/sites/default/files/01-Industry-Robotics-Intro.pdf.

5. Cfr. OIT, Perspectivas sociales y del empleo en el mundo - Tendencias 2016 (con datos referidos a 2015). Disponible en: http://www.ilo.org/wcmsp5/groups/public/_dgreports/—dcomm/_publ/documents 
6. El índice de malestar social para el período 2014-2015 experimenta una variación al alza del 6\% en los países desarrollados, mientras se mantiene en negativo para los países en desarrollo.

7. Cfr. http://www.enciclopediafinanciera.com/definicion-desempleo-estructural.html.

8. Cfr. N. Carr, The glass cage: Where automation is taking us. London, The Bodley Head, 2015; M. Ford, The lights in the tunnel: Automation, accelerating technology and the economy of the future. Charleston, CreateSpace Independent Publishing, 2009; M. Ford, The rise of the robots: Technology and the threat of a jobless future. New York, Basic Books, 2015; J. Kaplan, Humans need not apply: A guide to wealth and work in the age of artificial intelligence. New Haven, CT, Yale University Press, 2015; E. Brynjolfsson y A. McAfee, The second machine age: Work, progress, and prosperity in a time of brilliant technologies. WW Norton and Co, 2014.

9. Cfr. N. Agar, The sceptical optimist. Oxford, OUP, 2015.

10. Cfr. R. Kurzweil, The singularity is near: When humans transcend biology. London, Penguin, 2006.

11. Cfr. The Economist, 2 de abril de 2016. Disponible en: http://www.economist.com/news/business /21695908-silicon-valley-fights-talent-universities-struggle-hold-their?fsrc=scn/tw/te/pe/ed Imilliondollarbabies.

12. Piketty (cap. 9, nota 12) cita varias obras (Rebecca Blank, Changing Inequality. Berkeley, University of California Press, 2011; Raghuram Rajan, Fault Lines. Princeton, Princeton University Press, 2010) en las que detecta sesgos analíticos similares a los de Goldin/Katz; o carencias técnicas en el manejo de los datos, con muestras poco representativas.

13. Cfr. Patiño Rodríguez, David. "La incidencia del gasto público en educación sobre la distribución del bienestar económico". Hacienda Pública Española/Revista de Economía Pública 199, n. 4 (2011): 119-148; Calero Martínez, Jorge y María Gil Izquierdo. "La incidencia distributiva del gasto público en educación y sanidad en España". En 1er. Informe sobre la desigualdad en España. Madrid: Fundación Alternativas, 2013. (Citados por Goerlich Gisbert).

14. Cfr. Joan Rué, "El abandono universitario: variables, marcos de referencia y políticas de calidad". REDU. Revista de Docencia Universitaria, 12 (2), 2014: 281-306; Cabrera, L., Tomás, J., Álvarez, P. y Gonzalez, M., "El problema del abandono de los estudios universitarios", Relieve, no 12 (2), 2006: 171-203. Disponible en: http://www.uv.es/RELIEVE/v12n2/RELIEVEv12n2 1.htm;

15. Por alfabetización múltiple (multiliteracy) entiendo un conjunto de prácticas que incluyen la obtención, combinación, modificación, producción, presentación y evaluación de la información en diferentes formatos, entornos y situaciones, junto con la capacidad para seleccionar las herramientas $y$ metodologías adecuadas. La alfabetización múltiple requiere el desarrollo de las habilidades intelectuales necesarias para el aprendizaje y el pensamiento crítico, incluyendo la consideración de los aspectos éticos y estéticos involucrados en los problemas, así como el manejo del lenguaje y de los recursos expresivos necesarios para la construcción de conocimientos en las diferentes disciplinas (Halinen, Harmanen y Mattila 2015: 142-143).

16. Algunas aportaciones relevantes: Lucke y otros 2015, Racine y Forlini 2008, Lee y Silva 2009, Giroux, Parent y Guay 2010; Solomon, Noll y Mordkoff 2009; Turner y Sahakian 2006, Lucke y otros 2015, Alberini y Chen 2012; Lynch, Cox y Gall 2014.

17. Ordenadores portátiles, teléfonos móviles avanzados, tabletas o equipos informáticos de sobremesa con conexión permanente a redes digitales son la puerta de acceso a una variedad extraordinaria de productos de ocio y entretenimiento que constituyen la base de un proceso mucho más amplio de alfabetización tecnológica y digital, con un potencial infrautilizado en el contexto educativo.

18. Cfr., entre otros: C. POP, "Evaluation of E-learning Platforms: a Case Study", Informatica Economica, vol. 16 (1), 2012: 155-167. Disponible en: http://revistaie.ase.ro/content/61/14\%20-\%20Pop.pdf; F. Abbas Saeed, "Comparing and Evaluating Open Source E-learning Platforms". International Journal of Soft 
Computing and Engineering (IJSCE), Vol. 3 (3), 2013: 244-249. Disponible en: Comparing and Evaluating Open Source E-learning Platforms.pdf; S. Graf, B. List, "An Evaluation of Open Source E-Learning Platforms Stressing Adaptation Issues". Disponible en: https://moodle.org/other/icalt2005.pdf; G. Attwell (ed.), "Evaluating E-learning: A Guide to the Evaluation of E-learning". Evaluate Europe Handbook Series, vol. 2, 2006. Disponible en: http://pontydysgu.org/wp-content/uploads/2007/11 leva europe vol2 prefinal.pdf.

19. Los hay más especializados, como Hound; o con elementos más avanzados de inteligencia artificial, como Viv. Cfr. "Siri's inventors are building a radical new Al that does anything you ask", en:

http://www.wired.com/2014/08/viv; "10 Digital Assistant Alternatives To Apple's Sir", en: http://smallbiztrends.com/2014/05/10-digital-assistant-siri-alternatives.html; "Google Now, Siri And Cortana Shamed By New App", en: http://www.forbes.com/sites/jaymcgregor/2015/06/04/soundhoundvoice-assistant-hound/\#1bdf4887381f.

20. Existe ya una gran variedad de plataformas que ofrecen MOOCs. Cfr. https://es.wikipedia.org Iwiki/Mooc.

21. Cfr. http://noticias.universia.es/educacion/noticia/2013/11/25/1065271/6-plataformas-realizar-cursosmooc.html; http://formaciononline.eu/mooc-cursos-gratis-universidades;

http://blog.jobandtalent.com/universidades-que-imparten-cursos-gratuitos-online-mooc; $\quad$ https://ficod.es Imooc-o-como-estudiar-en-las-mejores-universidades-del-mundo-desde-casa.

22. Cfr. https://es.khanacademy.org; https://es.wikipedia.org/wiki/Khan Academy

(todos los enlaces indicados en las notas estaban activos el 15/05/2016).

\section{Bibliografía}

Alberini, Cristina M. (y Dillon Y. Chen)

2012 "Memory Enhancement: Consolidation, Reconsolidation and Insulin-like Growth Factor 2", Trends in Neurosciences, $\mathrm{n}^{\circ} 35$ (5): 274-83.

http://www.sciencedirect.com/science/article/pii/S0166223611002153.

Ansari, Daniel

2015 "Mind, Brain, and Education: A Discussion of Practical, Conceptual, and Ethical Issues", en Jens Clausen y Neil Levy (eds.), Handbook of Neuroethics. Dordrecht, Springer Netherlands: 1703-1719.

DOI: $10.1007 / 978-94-007-4707-4 \quad 146$

Au, Wayne W.

2008 "Devising Inequality, A Bernsteinian Analysis of High-stakes Testing and Social Reproduction in Education", British Journal of Sociology of Education, $\mathrm{n}^{\circ} 29$ (6): 639-651.

DOI: $10.1080 / 01425690802423312$.

Autor, David H.

2015 "Why Are There Still So Many Jobs? The History and Future of Workplace Automation", Journal of Economic Perspectives, $\mathrm{n}^{\circ} 29$ (3): 3-30.

DOI: $10.1257 /$ jep.29.3.3

Bähr, Sebastian (y Martin Abraham)

2016 "The Role of Social Capital in the Job-Related Regional Mobility Decisions of Unemployed Individuals", Social Networks, n 46 (July): 44-59.

DOI: 10.1016/j.socnet.2015.12.004

Berg, Maggie (y Barbara Seeber)

2016 The Slow Professor: Challenging the Culture of Speed in the Academy. Toronto, Buffalo, London, University of Toronto Press. 
Bertocchi, Graziella (y Michael Spagat)

2004 "The Evolution of Modern Educational Systems", Journal of Development Economics, $\mathrm{n}^{\circ} 73$ (2): 559-82.

DOI: $10.1016 / j . j d e v e c 0.2003 .05 .003$

Bian, Yanjie (y Xianbi Huang)

2015 "Beyond the Strength of Social Ties: Job Search Networks and Entry-Level Wage in Urban China", American Behavioral Scientist, $\mathrm{n}^{\circ} 59$ (8): 961-976.

DOI: $10.1177 / 0002764215580608$

Bozsik, Viola (coord.)

2015 Improving Literacy Skills across Learning: CIDREE Yearbook 2015. Budapest, HIERD.

Improving Literacy Skills Across Learning.pdf

Brynjolfsson, Erik (y Andrew McAfee)

2014 The Second Machine Age: Work, Progress, and Prosperity in a Time of Brilliant Technologies. NY, London, WW Norton and Co.

Chua, Vincent

2011 "Social Networks and Labour Market Outcomes in a Meritocracy", Social Networks, no 33 (1): 1-11.

DOI: 10.1016/j.socnet.2010.08.001

Cobo, Enrique

2010 "El abandono temprano de la educación y la formación en España", Revista de Educación, nº extr.:

31-62.

Danaher, John

2016 "Will Life Be Worth Living in a World Without Work? Technological Unemployment and the Meaning of Life", Science and Engineering Ethics, March.

DOI: $10.1007 / \mathrm{s} 11948-016-9770-5$

Daunton, Martin J.

1995 Progress and Poverty: An Economic and Social History of Britain, 1700-1850. $1^{\mathrm{a}}$ ed. Oxford, England, New York, Oxford University Press.

Delve, Janet

2007 "Joseph Marie Jacquard: Inventor of the Jacquard Loom", IEEE Annals of the History of Computing, $n^{\circ} 29$ (4): 98-102. Disponible en:

http://ieeexplore.ieee.org/stamp/stamp.jsp?tp=\&arnumber=4407450

Di Salvo, Philip

2013 "Revolutions between Digital Utopianism and the 'cascade Effect"', Studies in Communication Sciences, $\mathrm{n}^{\circ} 13$ (1): 103-104.

DOI: 10.1016/j.scoms.2013.04.009

Dirican, Cüneyt

2015 "The Impacts of Robotics, Artificial Intelligence On Business and Economics", Procedia - Social and Behavioral Sciences, no 195 (July): 564-573.

DOI: 10.1016/j.sbspro.2015.06.134

Donoghue, Frank

2008 The Last Professors: The Corporate University and the Fate of the Humanities. New York, Fordham University Press.

Freeth, Tony (y otros)

2006 "Decoding the Ancient Greek Astronomical Calculator Known as the Antikythera Mechanism", Nature, $\mathrm{n}^{\circ} 444$ (7119): 587-591.

DOI: $10.1038 /$ nature05357

Frey, Carl B. (y Michael A. Osborne) 
2013 The Future of Employment: How Susceptible Are Jobs to Computerisation? (Report). Disponible en: http://arche.depotoi.re/autoblogs/wwwinternetactunet 8a3fe3331e0ad7327e18d9fe6ec3f0ad04dcea58 Imedia/3722fa7d. The Future of Employment.pdf

García, Ana (y Cecilia Adrogué)

2015 "Abandono de los estudios universitarios: dimensión, factores asociados y desafíos para la política pública", Revista Fuentes, nº 16: 85-106.

DOI: 10.12795/revistafuentes.2015.i16.04

García, Carlos

1995 "Constantes y desafíos actuates de la profesion docente", Revista de Educación, n 306: 20-244.

Gerber, Larry

2014 The Rise and Decline of Faculty Governance: Professionalization and the Modern American University. Baltimore, John Hopkins University Press.

Giles, Jim

2013 "The truth behind the big data hype", New Scientist, nº 217 (2905): 48-49.

DOI: $10.1016 / \mathrm{S} 0262-4079(13) 60507-2$

Ginsberg, Benjamin

2013 The Fall of the Faculty: The Rise of the All-Administrative University and Why It Matters. Oxford, New York, Oxford University Press.

Giroux, S. (y otros)

2010 "La remédiation cognitive et la remédiation métacognitive pour les personnes ayant un TDAH: deux stratégies d'intervention novatrices et pourquoi pas complémentaires ?", Journal de Thérapie Comportementale et Cognitive, $\mathrm{n}^{\circ} 20$ (3): 87-92.

DOI: 10.1016/j.jtcc.2010.09.004

Goerlich, Francisco

2016 Distribución de la renta, crisis económica y políticas redistributivas. Bilbao, Fundación BBVA. Disponible en:

http://www.fbbva.es/TLFU/dat/DE 2016 IVIE Distribucion de la renta.pdf

Goldin, Claudia (y Lawrence F. Katz)

2010 The Race between Education and Technology: The Evolution of U.S. Educational Wage

Differentials. Cambridge, MA, Belknap Press.

Guereña, Jean-Louis (y Alejandro Tiana)

1989 Clases Populares, Cultura, Educación: Siglos XIX-XX. Madrid, Casa de Velázquez/UNED.

Halinen, Irmeli (y otros)

2015 "Making Sense of Complexity of the World Today: Why Finland Is Introducing Multiliteracy in Teaching and Learning", en Viola Bozsik (ed.), Improving Literacy Skills across Learning: CIDREE Yearbook 2015, Budapest, HIERD: 136-153. Disponible en:

http://www.oph.fi/download/173262_cidree_yb_2015_halinen_harmanen_mattila.pdf

He, Jackson (y Enrique Castro-León)

2009 "Automation Under Service-Oriented Grids", en Shimon Y. Nof (ed.), Springer Handbook of Automation. Berlin/Heidelberg, Springer: 405-416.

DOI: $10.1007 / 978-3-540-78831-7 \quad 24$

Jones, $\mathrm{S}$.

2006 Against technology: from the Luddites to Neo-Luddism. New York, Routledge.

Kottenstette, Nicholas (y Panos J. Antsaklis)

2009 "Communication in Automation, Including Networking and Wireless", en Shimon Y. Nof (ed.), Springer Handbook of Automation, Berlin/Heidelberg, Springer: 237-248.

DOI: $10.1007 / 978-3-540-78831-7 \quad 13$ 
Krueger, Dirk (y Krishna B. Kumar)

2004 "US-Europe Differences in Technology-Driven Growth: Quantifying the Role of Education", Journal of Monetary Economics, $\mathrm{n}^{\circ} 51$ (1): 161-190.

DOI: 10.1016/j.jmoneco.2003.07.005

Krug, Gerhard

2012 "(When) Is Job-Finding via Personal Contacts a Meaningful Concept for Social Network Analysis? A Comment to", Social Networks, nº 34 (4): 527-533.

DOI: 10.1016/j.socnet.2012.05.002

Lee, Yong-Seok (y Alcino J. Silva)

2009 "The Molecular and Cellular Biology of Enhanced Cognition", Nature Reviews. Neuroscience , n 10 (2): 126-140.

DOI:10.1038/nrn2572

Lucke, Jayne (y otros)

2015 "Using Neuropharmaceuticals for Cognitive Enhancement: Policy and Regulatory Issues", en Handbook of Neuroethics. Dordrecht, Springer Netherlands: 1085-1100.

DOI: $10.1007 / 978-94-007-4707-472$

Lynch, Gary (y otros)

2014 "Pharmacological Enhancement of Memory or Cognition in Normal Subjects", Frontiers in Systems Neuroscience, $\mathrm{n}^{\circ}$ 8: 90.

DOI: $10.3389 /$ fnsys. 2014.00090

Maher, Frances A. (y Mary K. Tetreault)

2008 "The Knowledge Economy and Academic Capitalism", British Journal of Sociology of Education, $\mathrm{n}^{\circ}$ 9 (6): $733-740$.

DOI: $10.1080 / 01425690802423726$

Morel, Gérard (y otros)

2009 "Reliability, Maintainability, and Safety", en Shimon Y. Nof (ed.), Springer Handbook of Automation.

Berlin/Heidelberg, Springer: 735-747.

DOI: $10.1007 / 978-3-540-78831-7 \quad 42$

Nau, Dana S.

2009 "Artificial Intelligence and Automation", en Shimon Y. Nof (ed.), Springer Handbook of Automation.

Berlin/Heidelberg, Springer: 249-268.

DOI: $10.1007 / 978-3-540-78831-7 \quad 14$

Newfield, Christopher

2008 Unmaking the Public University: The Forty-Year Assault on the Middle Class. Cambridge, Mass, Harvard University Press.

Nof, Shimon Y. (ed.)

2009 Springer Handbook of Automation. Berlin, Heidelberg, Springer.

DOI: $10.1007 / 978-3-540-78831-7$

Normand, Romuald

2008 "School Effectiveness or the Horizon of the World as a Laboratory", British Journal of Sociology of Education, n 29 (6): 665-676.

DOI: $10.1080 / 01425690802423346$

OIT

2015 Perspectivas sociales y del empleo en el mundo: Tendencias 2015. Disponible en:

http://www.ilo.org/wcmsp5/groups/public/—dgreports/_dcomm/_publ/documents/publication

IWCMs 337072.pdf

Piketty, Thomas

2014 Capital in the Twenty-First Century. Cambridge, Massachusetts, The Belknap Press of Harvard 
University Press.

Racine, Eric (y Cynthia Forlini)

2008 "Cognitive Enhancement, Lifestyle Choice or Misuse of Prescription Drugs?", Neuroethics, n 3 (1):

1-4.

DOI: $10.1007 / \mathrm{s} 12152-008-9023-7$

Rey, Olivier

2010 "The Use of External Assessments and the Impact on Education Systems", en Sheila M. Stoney (ed.), Beyond Lisbon 2010: Perspectives from Research and Development for Education Policy in Europe (CIDREE Yearbook 2010). Slough, NFER: 137-158. Disponible en:

The use of external assessments and the impact on education systems

Rifkin, Jeremy

1995 The End of Work: The Decline of the Global Labor Force and the Dawn of the Post-Market Era. New York, G. P. Putnam's Sons.

Robinson, Ken (y Lou Aronica)

2009 The Element: How Finding Your Passion Changes Everything. New York, Penguin Group USA.

Rué, Joan

2014 "El abandono universitario: variables, marcos de referencia y políticas de calidad", REDU. Revista de Docencia Universitaria, $\mathrm{n}^{\circ} 12$ (2): 281-306. Disponible en:

http://red-u.net/redu/index.php/REDU/article/view/506/pdf

Sandberg, Anders

2011 "Cognition Enhancement: Upgrading the Brain", en Julian Savulescu (y otros) (coord.), Enhancing Human Capacities. Chichester, West Sussex, U.K. Malden, MA, Wiley-Blackwell, 71-91.

Smith, Jayne

2008 "Quality Assurance and Gender Discrimination in English Universities: An Investigation", British Journal of Sociology of Education, $\mathrm{n}^{\circ} 29$ (6): 623-638.

DOI: $10.1080 / 01425690802423304$

Solomon, Louis M. (y otros)

2009 "Cognitive Enhancements in Human Beings", Gender Medicine, nº 6 (2): 338-344. Disponible en: http://www.sciencedirect.com/science/article/pii/S1550857909000758

Tiana, Alejandro (y otros)

1996 Secondary Education in Spain. Croton-on-Hudson, NY, Council of Europe Press Manhattan Pub. Co. distributor.

Turner, Danielle C. (y Barbara J. Sahakian)

2006 "Ethical Questions in Functional Neuroimaging and Cognitive Enhancement", Poiesis \& Praxis, n 4 (2): 81-94.

DOI: $10.1007 / \mathrm{s} 10202-005-0020-1$

Velásquez, Juan D. (y Shimon Y. Nof)

2009 "Collaborative E-Work, E-Business, and E-Service", en Shimon Y. Nof (ed.), Springer Handbook of Automation. Berlin, Heidelberg, Springer: 1549-1576.

DOI: $10.1007 / 978-3-540-78831-788$

Vernadat, François B.

2009 "Enterprise Integration and Interoperability", en Shimon Y. Nof (ed.), Springer Handbook of Automation. Berlin, Heidelberg, Springer: 1529-1538.

DOI: $10.1007 / 978-3-540-78831-7 \quad 86$

Wallen, Johanna

2008 The History of the Industrial Robot. Linkoping, Sweden. Disponible en:

http://liu.diva-portal.org/smash/get/diva2:316930/FULLTEXT01.pdf 
Wolte, Stefan C.

2010 "Efficiency in Education: 20 Years of Talk and No Progress?", en Sheila M. Stoney (ed.), Beyond Lisbon 2010: Perspectives from Research and Development for Education Policy in Europe (CIDREE Yearbook 2010). Slough, NFER: 197-210. Disponible en:

The use of external assessments and the impact on education systems

\section{World Economic Forum}

2016 The Future of Jobs. Employment, Skills and Workforce Strategy for the Fourth Industrial Revolution. Switzerland. Disponible en:

http://www3.weforum.org/docs/WEF Future of Jobs.pdf 